\title{
Metamaterial Sensor Platforms for Terahertz DNA Sensing
}

\author{
Nan Zheng, Student Member, IEEE, Mahdi Aghadjani, Kyungjun Song, and \\ Pinaki Mazumder, Fellow, IEEE
}

\begin{abstract}
Three high-sensitivity metamaterial Terahertz DNA sensors based on resonance are proposed to distinguish DNA molecule with different refractive indices. Both numerical electromagnetic method and physical circuit model interpretation are employed to analyze proposed sensor structures. Design guideline based on intuitive physical circuit model is provided and verified through full-wave simulation.
\end{abstract}

\section{INTRODUCTION}

Terahertz sensing has attracted numerous research activities in past decades. Due to the fact that many materials exhibit unique spectrum signatures in $\mathrm{THz}$ [1], [2], different materials can be easily characterized in this band. Although a lot of research are focused on wideband sensing that are trying to capture fingerprint of different materials in $\mathrm{THz}$ [3]-[5], narrow band $\mathrm{THz}$ bio-sensing based on resonance has been pursued extensively [6]-[12] as well since binding state of DNA can be identified through its refractive index change in $\mathrm{THz}$ [6]. Traditionally, the change in DNA state from the single-stranded DNA to the double-stranded DNA molecules is investigated by tagging the target DNA with certain agents such as fluorescent ones. Although this method has widespread applications in DNA sensing, it has disadvantages such as the unwanted interference from the tagging agent and the extra preparatory steps. For biomolecule like DNA, its refractive index depends on its binding state to known probes. Therefore measurement of the change in refractive index enables the label-free direct detection and identification of genetic sequences. In [7], [8], integrated on-chip microstrip line resonator is employed to sense refractive index change in DNA. A split ring resonator (SRR) on a paper substrate is demonstrated to have capability to distinguish glucose solutions with different concentrations [9].

Generally speaking, there are two important aspects in THz DNA resonance sensing. One is to increase sensitivity of the sensor. Various high Q resonators, such as asymmetric split ring resonators (ASRs) [10], $\Omega$-shaped resonator [11], are developed to achieve this. Another aspect is to reduce the amount of sample needed to characterize it. For example a near field source is employed in [12] to focus the energy onto a tiny spot beyond the diffraction limit, which consequently reduces the amount of sample needed.

This work is supported by the NSF under grant EAGER 1059177.

Nan Zheng, Mahdi Aghadjani and Pinaki Mazumder are with the Dept. of Electrical Engineering and Computer Science, University of Michigan, Ann Arbor, MI 48109 (e-mail: zhengn@umich.edu, mahdia@umich.edu, pinakimazum@gmail.com).

Kyungjun Song is with the Dept. of Nature-inspired Nano-converge System, Korea Institute of Machinery and Materials (e-mail: songk@kimm.re.kr).
In this paper, subwavelength scatterers are combined together to produce high order response that is useful to increase sensitivity of the DNA sensors. Three different sensing structures are proposed. Analysis is made based on their circuit models. Full-wave simulation results are also included to demonstrate performance of these sensors.

\section{SENSOR CONFIGURATION}

Configuration of bio-sensing platform in this paper is illustrated in Fig. 1. A metamaterial sensor platform shown in the figure consists of three layers: upper reactance layer, dielectric coupling layer and a lower reactance layer. Two reactance layers are composed by periodic subwavelength scatterers that are operating in metamaterial regime (i.e. its size is much smaller than the operating wavelength). Different from the traditional frequency selective surface (FSS) whose selectivity is based on capacitance and inductance provided by high order Floquet mode, this metamaterial sensor relies on the resonance characteristics of small scatterers. Some traditional FSSs can indeed have a very sharp pass-band or stop-band. Especially when the FSS operating near the Wood-Rayleigh anomaly, the extraordinary transmission takes place and an extremely sharp pass-band can occur [13]. Frequency of this extraordinary transmission, however, is mainly determined by the period of the scatterers instead of their individual feature. Therefore, metamaterial sensors based on resonant subwavelength scatterers will be more sensitive to the sample around it that can change its resonance feature.

When a sensing test is performed, a plane wave is incident normally onto the sensing platform, and a receiver is located at the other side to detect the transmission signal. Depending on types of small scatterers, a transmission peak, dip or both of them can occur. By putting samples with different refractive indices on the right location of the sensor, change in these transmission characteristics can be observed, which can help to distinguish different samples.

With such a periodic structure, electromagnetic response of a unit cell is enough to determine response of the entire sensor. In the following section, physical circuit model and numerical HFSS full-wave simulation are combined to analyze different sensor structure and provide a guide to design them. In the full-wave simulation, all metals and dielectrics are assumed to be perfect electric conductor and ideal dielectric without loss, respectively. Periodic boundary conditions are forced at four sides around the unit cell. Plane wave is illuminated from one of those two sides that are parallel with the sensor platform as shown in Fig. 1. And transmitted wave is detected from the other side. 


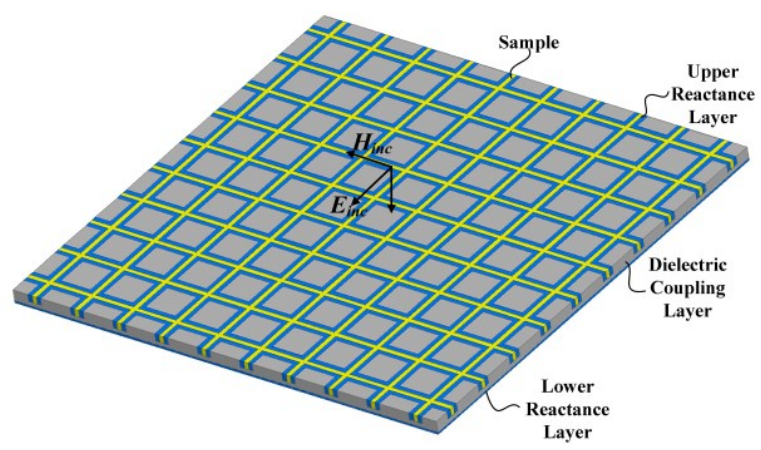

Figure 1. Configuration of metamaterial sensor.

\section{METAMATERIAL SENSORS}

\section{A. Patch-Wire Structure}

The first example is a patch-wire structure shown in Fig. 2(a) [14] whose equivalent circuit is shown in Fig. 2(b). Charge deposition along the slots between different patches provides the capacitance $C_{p}$, while current flow through wires contributes to the inductance $L_{w}$. An approximate equation to estimate $C_{p}$ and $L_{w}$ are shown in (1) and (2) [15].

$$
\begin{aligned}
& C_{p}=\varepsilon_{0} \varepsilon_{e f f} \frac{2 D_{p}}{\pi} \ln \left[\left(\sin \frac{\pi s_{p}}{2 D_{p}}\right)^{-1}\right] \\
& L_{w}=\mu_{0} \mu_{e f f} \frac{D_{w}}{2 \pi} \ln \left[\left(\sin \frac{\pi w_{w}}{2 D_{w}}\right)^{-1}\right]
\end{aligned}
$$

$Z_{0}$ and $Z_{d}$ are wave impedance in free space and dielectric, respectively. This equivalent circuit is valid when the patch layer and wire layer are infinitesimally thin. When thicknesses of these two layers are moderate, two series reactances are needed to represent influence of the thickness. However, their existence will not, at least qualitatively, influence our analysis much. Thus for the sake of simplicity, they are omitted in the equivalent circuit.

When the dielectric layer between patch and wire is electrically thin $(\theta<\pi / 6$, where $\theta$ is the electrical length of the dielectric layer) yet not too thin such that the patch and wire couple to each other directly, the short transmission line can be replaced by a $\Pi$ network as shown in Fig. 2(c). Furthermore, for thin dielectric layer, the shunt capacitors $C_{d}$ are so small compared to $C_{p}$ and $L_{w}$ that they can be neglected. By doing this, circuit in Fig. 2(d) is obtained. Obviously, a transmission maxima will occur when $C_{p}$ is parallel resonant with $L_{d}$ and $L_{w}$. Peak value of the pass-band is determined by the ratio of $L_{w}$ and $L_{d}$. A narrow pass-band can be obtained by employing small inductance and large capacitance. This can be easily justified through full-wave simulation results in Fig. 3. Small period of patch/wire means a small capacitance/inductance according to (1) and (2). As predicted before, a small inductance and a large capacitance configuration shown in Fig. 3(c) provides the narrowest passband. This merit is highly desired in bio-sensing because a narrow pass-band can make the change in $n$ more distinguishable. Besides, the lower transmission in Fig. 3(c) is caused by the fact that smaller $L_{w}$ can only draw less portion of power from the inductor divider. Simulation results are summarized in Table I for comparison.

To validate proposed circuit model, a comparison between results obtained by full-wave simulation and circuit model simulation is shown in Fig. 4. Apparently, the circuit model can qualitatively predict the transmission curve, providing a physical insight into the operation scheme of this sensor. The discrepancy between the two curves is mainly caused by the omitted series reactance that is determined by thickness of the reactance layer.

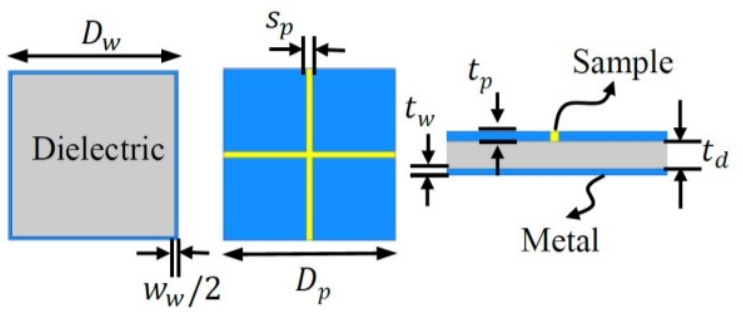

(a)

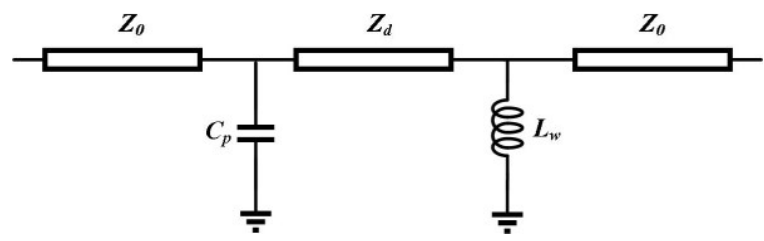

(b)

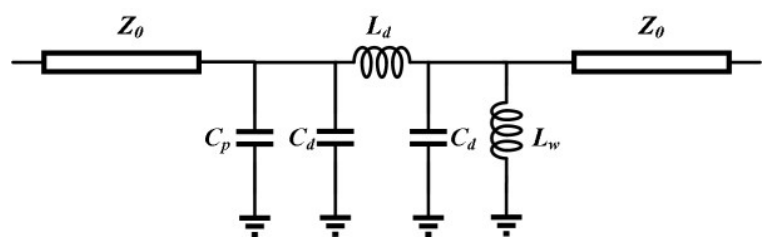

(c)

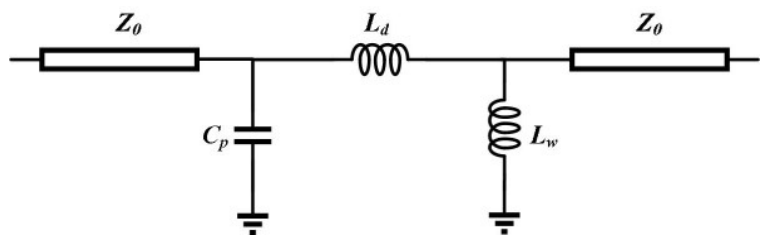

(d)

Figure 2. (a) Unit cell of patch-wire structure. (b) Circuit model of patchwire structure with dielectric coupling layer modeled as a short transmission line. (c) Circuit model with dielectric layer modeled as a $\Pi$ netowrk (d) Circuit model with shunt capacitors in (c) neglected.
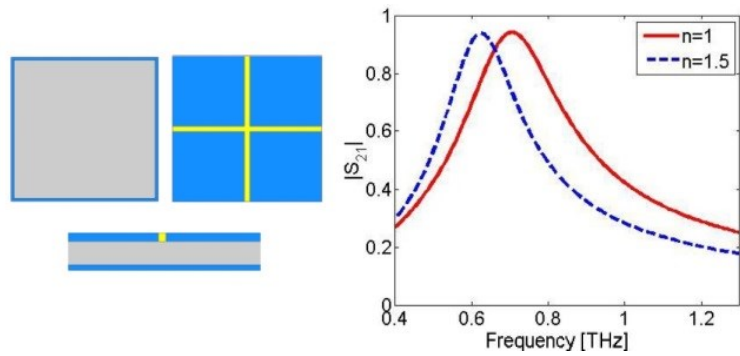

(a) 

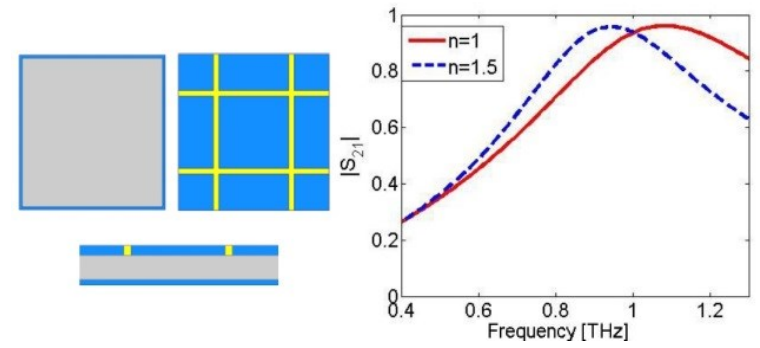

(b)
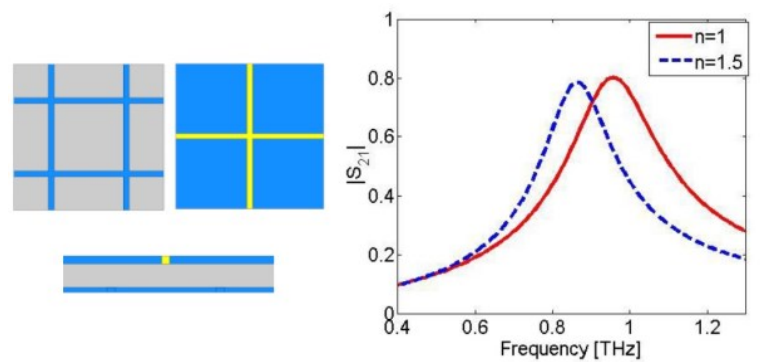

(c)

Figure 3. (a) Full-wave simulation results with dimensions: $D_{w}=46 \mu \mathrm{m}$, $D_{p}=46 \mu \mathrm{m}, t_{d}=4.4 \mu \mathrm{m}, t_{p}=1.5 \mu \mathrm{m}, t_{w}=1 \mu \mathrm{m}, s_{p}=1.7 \mu \mathrm{m}, w_{w}=1.7 \mu \mathrm{m}$. (b) Fullwave simulation results with dimensions: $D_{w}=46 \mu \mathrm{m}, D_{p}=23 \mu \mathrm{m}, t_{d}=4.4 \mu \mathrm{m}$, $t_{p}=1.5 \mu \mathrm{m}, t_{w}=1 \mu \mathrm{m}, s_{p}=1.7 \mu \mathrm{m}, w_{w}=1.7 \mu \mathrm{m}$. (c) Full-wave simulation results with dimensions: $D_{w}=23 \mu \mathrm{m}, D_{p}=46 \mu \mathrm{m}, t_{d}=4.4 \mu \mathrm{m}, t_{p}=1.5 \mu \mathrm{m}, t_{w}=1 \mu \mathrm{m}$, $s_{p}=1.7 \mu \mathrm{m}, w_{w}=1.7 \mu \mathrm{m}$.

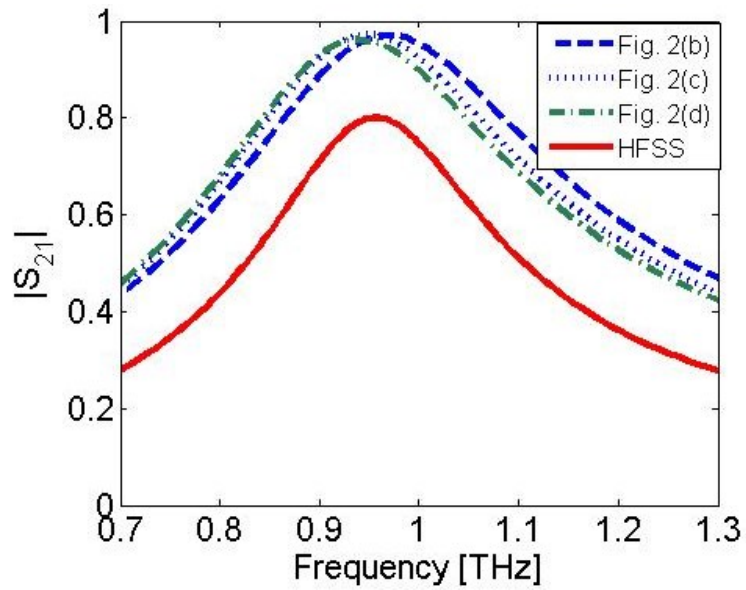

Figure 4. Comparison of simulation results between full-wave method and circuit models

TABLE I. RESULTS SUMMARY OF PATCH-WIRE STRUCTURES

\begin{tabular}{|l|c|c|c|c|}
\hline & $\begin{array}{c}\text { Resonant } \\
\text { Frequency } \\
\text { (THz) }\end{array}$ & $\begin{array}{c}\text { Relative } \\
\text { Resonant } \\
\text { Frequency Shift } \\
\left(\frac{\boldsymbol{f}_{\mathbf{n} 1}-\boldsymbol{f}_{\boldsymbol{n} \mathbf{2}}}{\boldsymbol{f}_{\boldsymbol{n} 1}}\right)\end{array}$ & $\begin{array}{c}\text { Relative 3-dB } \\
\text { Bandwidth } \\
\left(\frac{\boldsymbol{f}_{\boldsymbol{H}}-f_{\boldsymbol{L}}}{\boldsymbol{f}_{\mathbf{0}}}\right)\end{array}$ & $\left|\boldsymbol{S}_{\mathbf{2 1}}\right|$ \\
\hline Fig. 3 (a) & 0.705 & $11.3 \%$ & $34 \%$ & 0.941 \\
\hline Fig. 3 (b) & 1.085 & $12.1 \%$ & $67.2 \%$ & 0.960 \\
\hline Fig. 3 (c) & 0.955 & $10.6 \%$ & $22.5 \%$ & 0.800 \\
\hline
\end{tabular}

\section{B. Loop-Wire Structure}

In previous patch-wire structures, a huge capacitance is needed to narrow the pass-band. As can be seen from (1), one can either increase period of patch or decrease slot width to enhance capacitance. However, the period has to be controlled to be much smaller than the operating wavelength in order to stay in the metamaterial region; the slot width is also limited by the fabrication capability. One possible solution to this problem is to replace the patch layer with a loop layer as shown in Fig. 5 (a). The loop can be seen as a series branch composed of $C_{l}$ and $L_{l}[16]$. These capacitor and inductor are associated with the charge deposition between loop gap and current flowing along loop, respectively. The overall circuit model is shown in Fig. 5(b), where shunt capacitors brought by the transmission is again omitted. In the circuit model, a transmission zero is associated with the $L_{l}-C_{l}$ series branch. Close to this resonant point, this branch acts like a huge capacitance. This capacitance will parallel resonate with other reactance elements in the circuit to create a transmission maximum. It is this huge and frequencyvariant capacitance that we can exploit to sharpen the passband. Full-wave simulation results of this structure are shown in Fig. 6. Similar to the patch-wire structure, smaller inductance $L_{w}$ corresponds to a narrower pass-band. The lower transmission peak is caused by the same reason as in the patch-wire structure: a smaller $L_{w}$ can only draw less power from the inductor divider. Table II lists simulation results for loop-wire structures.

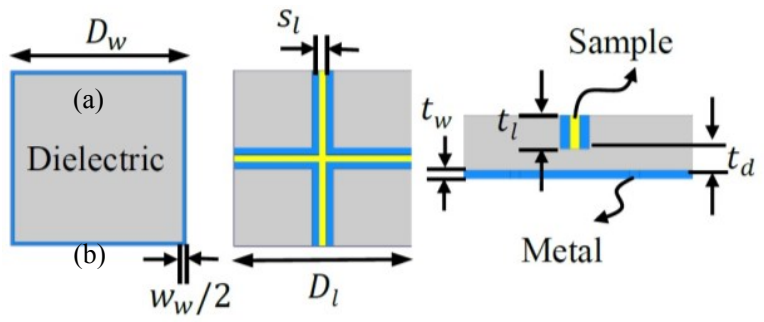

(a)

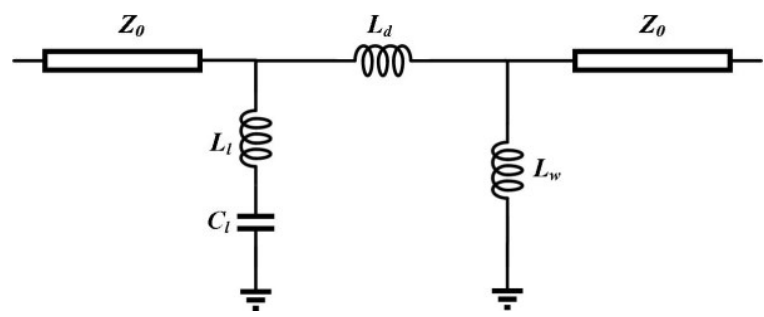

(b)

Figure 5. (a) Unit cell of loop-wire structure. (b) Circuit model of loopwire structure.
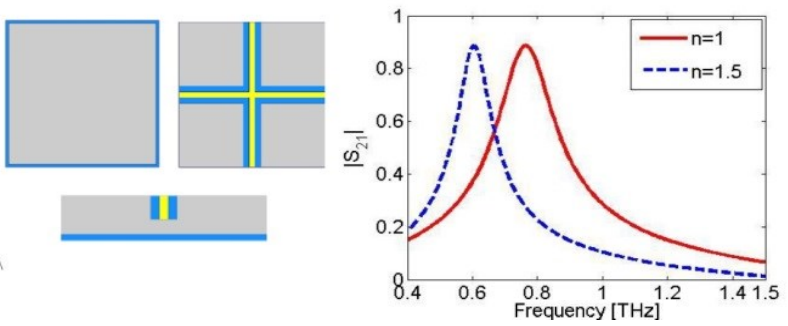

(a) 

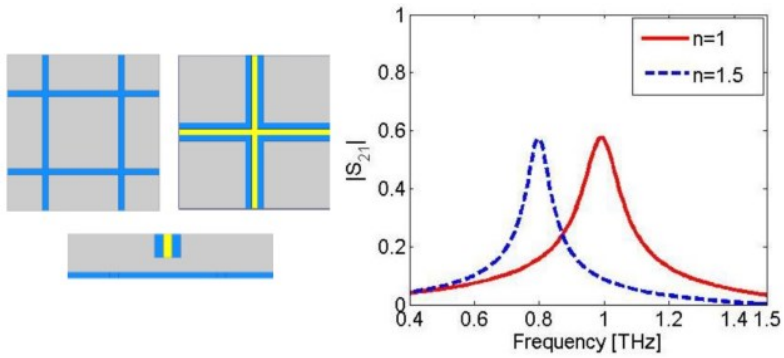

(b)

Figure 6. (a) Full-wave simulation results with dimensions: $D_{w}=27 \mu \mathrm{m}$, $D_{l}=27 \mu \mathrm{m}, t_{d}=2.8 \mu \mathrm{m}, t_{l}=4.4 \mu \mathrm{m}, t_{w}=1.1 \mu \mathrm{m}, s_{l}=1.1 \mu \mathrm{m}, w_{w}=2.2 \mu \mathrm{m}$. (b) Fullwave simulation results with dimensions: $D_{w}=13.5 \mu \mathrm{m}, D_{l}=27 \mu \mathrm{m}$, $t_{d}=2.8 \mu \mathrm{m}, t_{l}=4.4 \mu \mathrm{m}, t_{w}=1.1 \mu \mathrm{m}, s_{l}=1.1 \mu \mathrm{m}, w_{w}=2.2 \mu \mathrm{m}$

TABLE II. RESULTS SUMMARY OF LOOP-WIRE STRUCTURES

\begin{tabular}{|l|l|l|l|l|}
\hline & $\begin{array}{c}\text { Resonant } \\
\text { Frequency } \\
\text { (THz) }\end{array}$ & $\begin{array}{c}\text { Relative } \\
\text { Resonant } \\
\text { Frequency } \\
\text { Shift }\left(\frac{f_{n 1}-\boldsymbol{f}_{\boldsymbol{n} 2}}{\boldsymbol{f}_{\boldsymbol{n} 1}}\right)\end{array}$ & $\begin{array}{c}\text { Relative 3-dB } \\
\text { Bandwidth } \\
\left(\frac{f_{\boldsymbol{H}}-\boldsymbol{f}_{\boldsymbol{L}}}{\boldsymbol{f}_{\mathbf{0}}}\right)\end{array}$ & $\left|\boldsymbol{S}_{\mathbf{2 1}}\right|$ \\
\hline Fig. 6 (a) & 0.765 & $17.6 \%$ & $35.9 \%$ & 0.886 \\
\hline Fig. 6 (b) & 0.99 & $19.6 \%$ & $16.6 \%$ & 0.576 \\
\hline
\end{tabular}

\section{Symmetrical Loop Structure}

One common yet minor drawback in patch-wire and loopwire structures is that at the output stage, there is always an inductor divider to prevent all power being transferred. In order to sharpen the pass-band, a smaller $L_{w}$ is needed. This will, however, decrease output power. It seems feasible to decrease coupling inductance $L_{d}$ associated with the dielectric layer between two reactance layers to solve this problem. This, however, has two problems. One is that thickness of dielectric layer is limited by the fabrication process. Moreover, when the dielectric is too thin, direct electromagnetic coupling may occur between these two reactance layers, which is not expected in our case.

One solution to this problem is a symmetrical loop structure shown in Fig. 7 (a). Two identical loops are separated by a dielectric coupling layer. Its circuit model is shown in Fig. 7(b). One obvious observation is that there is a transmission zero provided by the resonance of loop. Due to symmetry of this structure, an even-odd mode analysis is used to split the circuit into even and odd mode as shown in Fig. 7(c) and Fig. 7(d). Transmission coefficient of this circuit can be obtained in (3) through basic circuit theory.

$$
S_{21}=\frac{1}{2} \Gamma_{e}-\frac{1}{2} \Gamma_{o}
$$

where

$$
\begin{gathered}
\Gamma_{e}=\frac{Z_{e}-Z_{0}}{Z_{e}+Z_{0}}, \Gamma_{o}=\frac{Z_{o}-Z_{0}}{Z_{o}+Z_{0}}, \\
Z_{e}=j \omega L_{l}-\frac{j}{\omega C_{l}}, Z_{o}=\frac{1}{\frac{1}{j \omega L_{d} / 2}+\frac{1}{Z_{e}}}
\end{gathered}
$$

In (3), $S_{21}$ is the transmission coefficient from input to output; $\Gamma_{e}\left(\Gamma_{o}\right), Z_{e}\left(Z_{o}\right)$ are the reflection coefficient and the input impedance at output port for the even(odd) mode circuit, respectively. If the parallel resonance frequency of odd mode circuit $f_{o}$ is not far away from even mode circuit resonance frequency $f_{e}$, then $\Gamma_{o}$ is 1 and $\Gamma_{e}$ is around -1 at $f_{o}$ since $Z_{e}$, in this case, is much smaller than $Z_{0}$. Consequently, a transmission peak with a $100 \%$ power being transmitted can be achieved. Even when $f_{o}$ and $f_{e}$ is separated, there will always be a frequency below $f_{o}$ at which $Z_{e}$ is capacitive and $Z_{o}$ is inductive such that $\Gamma_{o}$ is $-\Gamma_{e}$. Subsequently, $S_{2 l}$ is 1 . Fullwave simulation result for this structure is shown in Fig. 8 (a). With this structure, samples are needed to be filled within the gaps between loops on both sides. An appropriate support to the samples on the backside is assumed here.

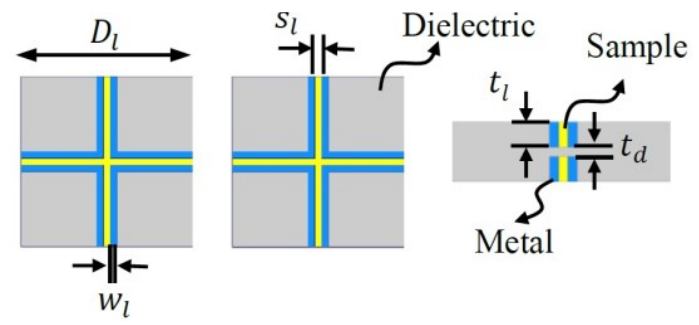

(a)

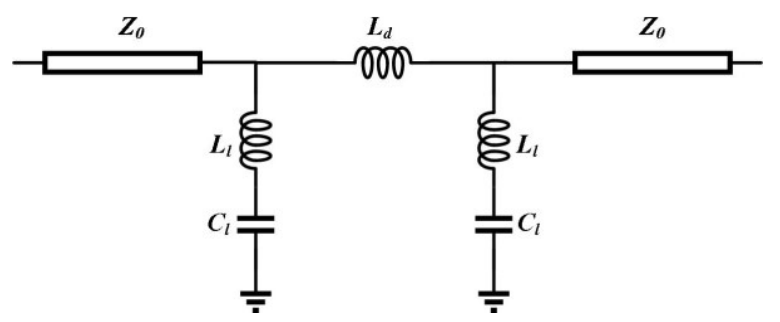

(b)

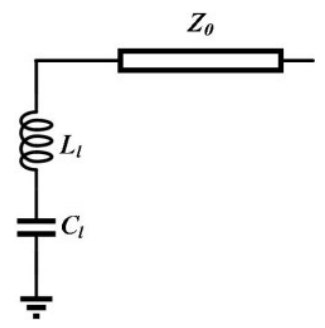

(c)

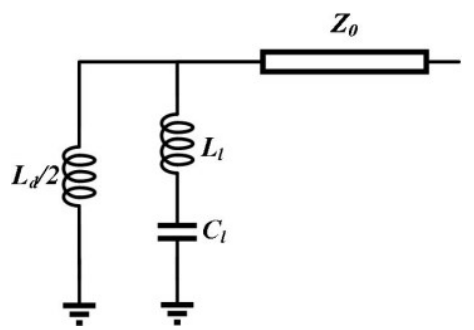

(d)
Figure 7. (a) Unit cell of symmetrical loop structure. (b) Circuit model of symmetrical loop structure. (c) Even mode circuit of the circuit model in (b). (d) odd mode circuit of the circuit model in (b).

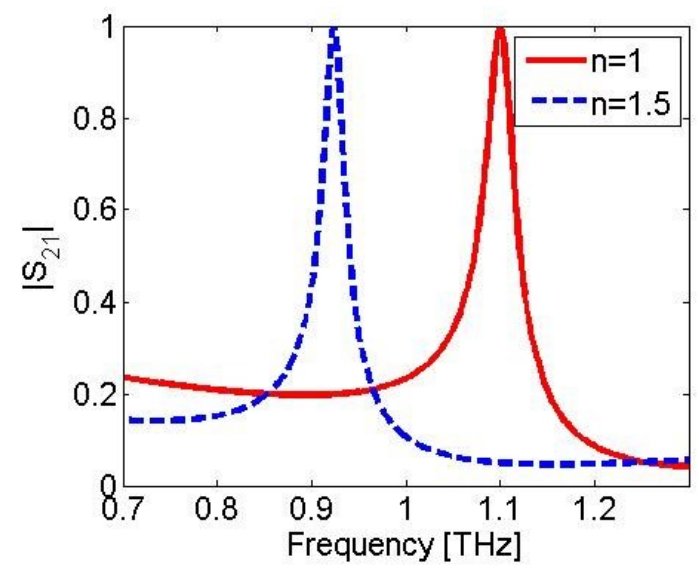

(a) 


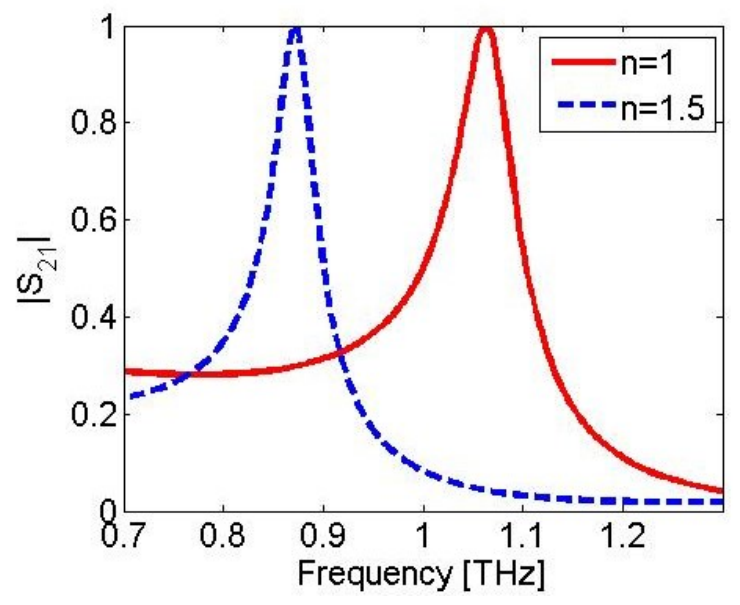

(b)

Figure 8. (a) Full-wave simulation results with dimensions: $D_{l}=37.5 \mu \mathrm{m}$, $t_{d}=2.25 \mu \mathrm{m}, t_{l}=6 \mu \mathrm{m}, s_{l}=1.5 \mu \mathrm{m}, w_{l}=1.5 \mu \mathrm{m}$. (b) Full-wave simulation results with dimensions: $D_{l}=30 \mu \mathrm{m}, t_{d}=5.4 \mu \mathrm{m}, t_{l}=4.8 \mu \mathrm{m}, s_{l}=1.2 \mu \mathrm{m}, w_{l}=1.2 \mu \mathrm{m}$.

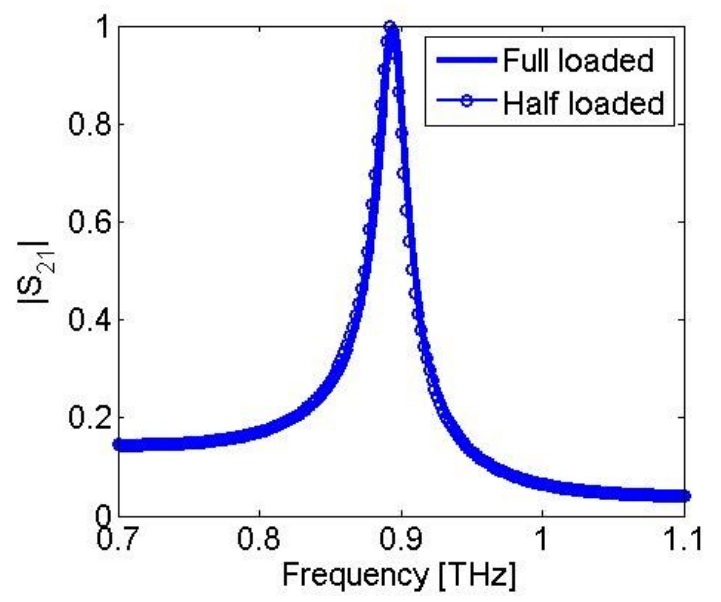

Figure 9. Comparison of results obtained from sensor with all slots loaded with sample and sensor with only half slots are loaded.

One interesting feature of this symmetrical loop structure is that resonance frequency shift can be controlled by deliberate design. In this structure, the more $f_{o}$ is deviated from $f_{e}$, the further away $f_{c t}$ is located below $f_{o}$ since a smaller $Z_{o}$ is needed. Thus one can increase thickness of substrate to enhance $L_{d}$, which then provides a larger separation between $f_{e}$ and $f_{o}$ that can gives rise to a larger frequency shift. Of course, a larger $L_{d}$ will, according to the circuit model, widen the pass-band. Therefore, a tradeoff can be made between narrow bandwidth and large resonance frequency shift. To illustrate this, simulation results of increasing $L_{d}$ is shown in Fig. 8(b) with other dimensions also being modified to make it resonates at the same frequency. Simulation results are summarized in Table III for the purpose of comparison.

In addition, the amount of sample needed in a test can be reduced by half if one uses an appropriate polarization of incident wave. Only slots perpendicular to the electric field needs to be filled since electric field mainly interacts with these slots. Fig. 9 plots the transmission curves obtained from sensor with all the slots filled and sensor with only slots perpendicular to the electric field polarization direction filled. Apparently, there is no practical difference between these two curves. Therefore, amount of sample needed is reduced.

TABLE III. RESULTS SUMMARY OF SYMMETRICAL LOOP STRUCTURE

\begin{tabular}{|c|l|l|l|l|}
\hline & $\begin{array}{c}\text { Resonant } \\
\text { Frequency } \\
\text { (THz) }\end{array}$ & $\begin{array}{c}\text { Relative } \\
\text { Resonant } \\
\text { Frequency } \\
\text { Shift }\left(\frac{\boldsymbol{f}_{\boldsymbol{n} 1}-\boldsymbol{f}_{\boldsymbol{n} 2}}{\boldsymbol{f}_{\boldsymbol{n} 1}}\right)\end{array}$ & $\begin{array}{c}\text { Relative 3-dB } \\
\text { Bandwidth } \\
\left(\frac{\boldsymbol{f}_{\boldsymbol{H}}-\boldsymbol{f}_{\mathbf{L}}}{\boldsymbol{f}_{\mathbf{0}}}\right)\end{array}$ & $\left|\boldsymbol{S}_{\mathbf{2 1}}\right|$ \\
\hline Fig. 8 (a) & 1.102 & $16.0 \%$ & $2.5 \%$ & 0.986 \\
\hline Fig. 8 (b) & 1.062 & $17.8 \%$ & $3.3 \%$ & 0.999 \\
\hline
\end{tabular}

\section{CONCLUSION}

In summary, three different $\mathrm{THz}$ metamateral DNA sensors are proposed. Design guideline based on circuit model counterparts is outlined in the paper. Full-wave simulation results validate the high sensitivity achieved by proposed sensors.

\section{REFERENCES}

[1] Withayachumnankul, W.; Png, G.M.; Xiaoxia Yin; Atakaramians, S.; Jones, I.; Hungyen Lin; Seam Yu Ung; Balakrishnan, J.; Ng, B.W.-H.; Ferguson, B.; Mickan, S.P.; Fischer, B.M.; Abbott, D.; , "T-Ray Sensing and Imaging," Proceedings of the IEEE, vol.95, no.8, pp.1528-1558, Aug. 2007

[2] O'Hara, J. F., Withayachumnankul, W, Al-Naib, I. (2012). A review on thin-film sensing with terahertz waves. Journal of Infrared, Millimeter and Terahertz Waves, 1-47.

[3] Fischer, B.M.; Helm, H.; Jepsen, P.U.; , "Chemical Recognition With Broadband THz Spectroscopy," Proceedings of the IEEE, vol.95, no.8, pp.1592-1604, Aug. 2007

[4] J. Zhang, and D. Grischkowsky, "Waveguide terahertz time-domain spectroscopy of nanometer water layers," Optics Letters, vol. 29, no. 14 , pp. $1617-1619,2004$

[5] M. B. Byrne, J. Cunningham, K. Tych, A. D. Burnett, M. R. Stringer, C. D. Wood, L. Dazhang, M. Lachab, E. H. Linfield, and A. G. Davies, "Terahertz vibrational absorption spectroscopy using microstrip-line waveguides," Applied Physics Letters, vol. 93, no. 18, p. 182904, 2008.

[6] M. Brucherseifer, M. Nagel, P. Haring Bolivar, H. Kurz, A. Bosserhoff, and R. Buettner, "Label-free probing of the binding state of DNA by time-domain terahertz sensing," Applied Physics Letters, vol. 77, no. 24, pp. 4049-4051, 2000.

[7] M. Nagel, P. Haring Bolivar, M. Brucherseifer, H. Kurz, A. Bosserhoff, and R. Buettner, "Integrated THz technology for labelfree genetic diagnostics," Applied Physics Letters, vol. 80, no. 1, pp. 154-156, 2002.

[8] P. H. Bolivar, M. Nagel, F. Richter, M. Brucherseifer , H. Kurz, A. Bosserhoff and R. Buttner "Label-free $\mathrm{THz}$ sensing of genetic sequences: Towards 'THz biochips' ", Philos. Trans. R. Soc. London Ser. A Math. Phys. Eng. Sci., vol. 362, pp.323 -333 2004

[9] H. Tao, L. Chieffo, M. A. Brenckle, S. M. Siebert, M. Liu, A. C. Strikwerda, K. Fan, D. L. Kaplan, X. Zhang, R. D. Averitt, and F. G. Omenetto, "Metamaterials on paper as a sensing platform," Advanced Materials, vol. 23, pp. 3197-3201, 2011.

[10] V. Fedotov, M. Rose, S. Prosvirnin, N. Papasimakis, and N. Zheludev, "Sharp trapped-mode resonances in planar metamaterials with a broken structural symmetry," Physical Review Letters, vol. 99, p. 147401, 2007.

[11] M. Huang, J. Yang, J. Sun, J. Shi, and J. Peng, "Modelling and analysis of $\Omega$-shaped double negative material-assisted microwave sensor,"Journal of Infrared, Millimeter, and Terahertz Waves, vol. 30 , no. 11, pp. 1131-1138, 2009. 
[12] W. Withayachumnankul, H. Lin, K. Serita, C. Shah, S. Sriram, M. Bhaskaran, M. Tonouchi, C. Fumeaux, and D. Abbott, "Subdiffraction thin-film sensing with planar terahertz metamaterials," Optics Express, vol. 20, no. 3, pp. 3345-3352, 2012.

[13] Medina, F.; Mesa, F.; Marques, R , "Extraordinary Transmission Through Arrays of Electrically Small Holes From a Circuit Theory Perspective," Microwave Theory and Techniques, IEEE Transactions on, vol.56, no.12, pp.3108-3120, Dec. 2008.

[14] Sarabandi, K.; Behdad, N , "A Frequency Selective Surface With Miniaturized Elements," Antennas and Propagation, IEEE Transactions o, vol.55, no.5, pp.1239-1245, May 2007.

[15] Al-Joumayly, M.A.; Behdad, N.; , "A Generalized Method for Synthesizing Low-Profile, Band-Pass Frequency Selective Surfaces With Non-Resonant Constituting Elements," Antennas and Propagation, IEEE Transactions on, vol.58, no.12, pp.4033-4041, Dec. 2010.

[16] Monacelli, B.; Pryor, J.B.; Munk, B.A.; Kotter, D.; Boreman, G.D.; , "Infrared frequency selective surface based on circuit-analog square loop design," Antennas and Propagation, IEEE Transactions on, vol.53, no.2, pp. 745- 752, Feb. 2005. 